\title{
Autonomic dysfunction in chronic inflammatory demyelinating polyradiculoneuropathy
}

J.J. Figueroa, MD

P.J.B. Dyck, MD

R.S. Laughlin, MD

J.A. Mercado, MD

R. Massie, MD

P. Sandroni, MD, PhD

P.J. Dyck, MD

P.A. Low, MD

Correspondence \& reprint requests to Dr. Low:

low@mayo.edu

\section{ABSTRACT}

Objectives: Autonomic deficits in chronic inflammatory demyelinating polyradiculoneuropathy (CIDP) have not been adequately quantitated. The Composite Autonomic Severity Score (CASS) is a validated instrument for laboratory quantitation of autonomic failure derived from standard autonomic reflex tests. We characterized dysautonomia in CIDP using CASS.

Methods: Autonomic function was retrospectively analyzed in 47 patients meeting CIDP criteria. CASS ranges from 0 (normal) to 10 (pandysautonomia), reflecting summation of sudomotor (0-3), cardiovagal (0-3), and adrenergic (0-4) subscores. Severity of neurologic deficits was measured with Neuropathy Impairment Score (NIS). Degree of small fiber involvement was assessed with quantitative sensation testing. Thermoregulatory sweat test (TST) was available in 8 patients.

Results: Patients ( 25 men) were middle-aged ( $45.0 \pm 14.9$ years) with longstanding CIDP (3.5 \pm 4.3 years) of moderate severity (NIS, $46.5 \pm 32.7)$. Autonomic symptoms were uncommon, mainly gastrointestinal $(9 / 47 ; 19 \%)$ and genitourinary $(8 / 47 ; 17 \%)$. Autonomic deficits (CASS $\geq 1$ ) were frequent $(22 / 47 ; 47 \%)$ but very mild (CASS, $0.8 \pm 0.9 ;$ CASS $\leq 3$, all cases). Deficits were predominantly sudomotor $(16 / 47 ; 34 \%)$ and cardiovagal $(10 / 47 ; 21 \%)$ with relative adrenergic sparing (4/47; 9\%). TST was abnormal in 5 of 8 patients (anhidrosis range, $2 \%-59 \%$ ). Sudomotor impairment was predominantly distal and postganglionic. Somatic deficits (disease duration, severity, small fiber deficits) did not predict presence of autonomic deficits.

Conclusion: Our data characterize the autonomic involvement in classic CIDP as mild, cholinergic, and predominantly sudomotor mainly as a result of lesions at the distal postganglionic axon. Extensive or severe autonomic involvement $(C A S S \geq 4)$ in suspected CIDP should raise concern for an alternative diagnosis. Neurology ${ }^{\circledR}$ 2012;78:702-708

\section{GLOSSARY}

AIDP = acute inflammatory demyelinating polyradiculoneuropathy; ARS = autonomic reflex screen; CASS = Composite Autonomic Severity Score; CIDP = chronic inflammatory demyelinating polyradiculoneuropathy; DLRPN = diabetic lumbosacral radiculoplexus neuropathy; HUT = head-up tilt; NIS = Neuropathy Impairment Score; NSC = Neuropathy Symptoms and Change Score; QSART = quantitative sudomotor axon reflex testing; QST = quantitative sensation testing; TST = thermoregulatory sweat test.

Involvement of somatic nerve fibers in chronic inflammatory demyelinating polyradiculoneuropathy (CIDP) has been characterized as affecting large myelinated fibers more than small myelinated and unmyelinated fibers. However, involvement of autonomic fibers has not been well characterized. Studies on dysautonomia in CIDP report variable prevalence of autonomic deficits $(21 \% \text { to } 76 \%)^{1-4}$ probably due to different sample sizes and inclusion of autonomic tests with low sensitivity, specificity, reproducibility, or unknown confounding variables. Large series on CIDP report infrequent and mild autonomic complaints. ${ }^{5-7}$ However, severe dysautonomia has been described in anecdotal reports. ${ }^{8-11}$ Quantification of autonomic failure using validated instruments is lacking in CIDP. The Composite Autonomic Severity Score (CASS) is an instrument for laboratory quantification of autonomic failure into a single validated score that corrects for the confounding effects of age and gender. ${ }^{12}$

The aim of this study was to characterize the autonomic dysfunction in patients with CIDP using CASS. Specifically we assessed 1) frequency, severity, and distribution of autonomic

From the Department of Neurology, Mayo Clinic, Rochester, MN.

Study funding: Funding information is provided at the end of the article.

Disclosure: Author disclosures are provided at the end of the article. 
deficits; 2) the potential site of the autonomic lesion; and 3) whether autonomic deficits related to somatic neurologic deficits. We hypothesized that 1) some degree of autonomic dysfunction is present; 2) the site of the autonomic lesion is mostly distal, reflecting summated distal axonal dysfunction, with some cases demonstrating a ganglionopathy or proximal nerve involvement; and 3) autonomic dysfunction is associated with disease duration and severity or somatic small nerve fiber involvement. Combining a variety of autonomic testing into a single validated scale will lead to better characterization of autonomic dysfunction in CIDP.

METHODS Standard protocol approvals, registrations, and patient consents. After approval from the appropriate institutional review board, consenting medical records were reviewed.

We identified medical records of cases with a potential diagnosis of CIDP with full neurophysiologic and neurologic evaluation performed between 1992 and 2009 using the central diagnostic index at Mayo Clinic. A comprehensive list of keywords that encompassed diagnoses and syndromes related to CIDP were used (e.g., Guillain-Barré syndrome, polyradiculopathy). Those who underwent autonomic reflex screen (ARS) were further identified by review of Mayo Autonomic Laboratory records. The ARS is routinely performed in the vast majority of patients evaluated for peripheral neuropathy at our institution. Thermoregulatory sweat test (TST) was not required to avoid selection bias on the assumption that more severely affected or atypical patients are more likely to undergo TST.

Only patients with classic CIDP with a negative workup for conditions associated with neuropathy were included in the analysis ( $\mathrm{n}=47$ cases). Autonomic symptoms were retrospectively abstracted from systematic chart review by only one author (J.F.).

After chart review was completed, we retrospectively identified a subset of patients who had been enrolled in neuropathy research studies following their initial clinical visit for neurologic complaints. In this subgroup of patients, symptoms had been quantitated with the Neuropathy Symptoms and Change Score (NSC), which has an autonomic questionnaire subcomponent. We used these cross-sectional data for internal validation of data obtained through chart review.

Because we did not use demographic selection criteria, our study population was not limited to those living relatively close to Mayo Clinic Rochester, i.e., Olmsted County. Hence, our study population reflected the combined local and referral population.

Inclusion criteria. Patients were included if they met 3 clinical criteria for classic CIDP as originally described by Dyck et al. ${ }^{5}$ and further modified by Laughlin et al. ${ }^{13}: 1$ ) initial clinical deterioration over a period of $\geq 8$ weeks with subsequent monophasic, progressive, or relapsing course; 2) preferential or at least equal involvement of large nerve fibers (motor or vibration and proprioception) as compared to small sensory (pain and temperature) or autonomic fibers; and 3) symmetric involvement of proximal and distal segments of all 4 limbs with hyporeflexia or areflexia. After fulfillment of all clinical criteria, patients were then required to show strong evidence of a demyelinating process by meeting one of 3 possible sets of electrophysiologic criteria: 1) definite American Academy of Neurology AIDS Task Force CIDP electrophysiologic criteria $\left.{ }^{14}(\mathrm{n}=30) ; 2\right)$ definite European Federation of Neurological Societies/Peripheral Nerve Society electrophysiologic criteria ${ }^{15}$ plus supportive evidence defined as response to immune therapy or CSF albuminocytologic dissociation ( $n=16)$; or 3 ) probable electrophysiologic criteria by either group plus nerve biopsy showing segmental demyelination and prominent onion bulbs $(\mathrm{n}=1)$.

Exclusion criteria. To restrict analysis to classic forms of CIDP, patients were excluded if they had 1) refractory CIDP or atypical variants of CIDP (e.g., pure sensory, pure motor, pure distal, multifocal, asymmetric, or localized forms); 2) mechanical intrathecal compression due to massive nerve root hypertrophy resulting in myelopathy, cauda equina syndrome, or lumbosacral spinal canal stenosis; 3) coexistent conditions that suggest secondary CIDP (e.g., HIV, multiple myeloma, sarcoid, Crohn disease, Sjögren disease); 4) other causes of demyelination that can potentially mimic CIDP (e.g., monoclonal gammopathy, hereditary demyelinating neuropathies, POEMS, diabetic lumbosacral radiculoplexus neuropathy); 5) alternative causes of neuropathy (e.g., diabetes, paraneoplasia, B12 deficiency); and 6) other causes of autonomic dysfunction (e.g., spondylitic myelopathy, multiple sclerosis) including failure to properly discontinue (5 half-lives) medications that affect autonomic function prior to autonomic testing.

Neuropathy Impairment Score. The Neuropathy Impairment Score (NIS) is a standardized measurement of severity of somatic neuropathic deficits, both motor and sensory. ${ }^{16}$ Muscle weakness of a predetermined group of muscles, loss of deep tendon reflexes in upper and lower limbs, and loss of large and small fiber sensation of index fingers and great toes are summated and expressed as a composite score ranging from 0 (no impairment) to 244 (maximal impairment). The examiner grades abnormality of each item according to sex, age, physical fitness, and other anthropomorphic characteristics.

Quantitative sensation testing. Computer-assisted sensory examination (CASE IV) of the lower limb was performed to establish the type of somatic sensory fiber involvement. ${ }^{17,18}$ The magnitude of the stimulus to detect vibration (large myelinated), cooling (small myelinated), and heat-pain (small unmyelinated) was measured and expressed as a percentile of a normal population of the same age and sex. Vibratory, cooling, and intermediate heat-pain (HP 5.0) thresholds were considered abnormal when $\geq 95$ th percentile for any modality (hypoesthesia or hypoalgesia) or $\leq 5$ th percentile for heat-pain (hyperalgesia).

Neuropathy Symptoms and Change Score. The NSC is a standardized measurement of number, severity, and change in motor, sensory, and autonomic neuropathic symptoms. ${ }^{19}$ The autonomic component is comprised by a questionnaire for the presence of symptoms related to orthostatic intolerance, upper and lower gastrointestinal dysmotility, genitourinary dysfunction, rectal sphincter control, and sicca complaints.

Autonomic Reflex Screen. The ARS consists of a set of autonomic function tests routinely used in clinical practice to assess the severity and distribution of sudomotor, cardiovagal, and adrenergic deficits and have been previously described. ${ }^{20}$ Quantitative sudomotor axon reflex test (QSART) at proximal and distal standard sites was used to assess the integrity of the sympathetic postganglionic sudomotor axon and the pattern of involvement (e.g., length-dependent). ${ }^{21}$ Beat-to-beat heart rate responses to 
deep breathing and the Valsalva maneuver were used as indices of cardiovagal function. Beat-to-beat blood pressure responses to the Valsalva maneuver and head-up tilt (HUT) were used as indices of adrenergic function. ${ }^{20}$

Thermoregulatory sweat test. The TST assesses the integrity of central and peripheral sudomotor pathways involved in the thermoregulatory response to changes in environmental temperature. $^{22}$ TST characterizes the distribution of sweat loss, provides a quantitative measure of anterior body surface anhidrosis, and when combined with QSART, helps in establishing the site of lesion relative to the autonomic ganglia (preganglionic vs postganglionic). ${ }^{23,24}$ Sweating is demonstrated by a change in color of an indicator powder applied on the surface of a patient lying supine inside a sweat cabinet. The percent of anhidrosis in the anterior body surface is calculated from digital photographs. ${ }^{22}$ Results are expressed as percent of anterior body surface anhidrosis and patterns of anhidrosis (i.e., distal, focal, segmental, regional, global, and mixed). In addition, at QSART sites, lesions can be categorized as 1) "preganglionic" if TST shows anhidrosis but corresponding QSART is normal; and 2) "postganglionic" if QSART is abnormal.

Composite Autonomic Severity Score. The CASS has been validated to provide a measure of severity and distribution of autonomic failure and is derived from the autonomic reflex screen as previously described. ${ }^{12}$ The CASS score ranges from 0 to 10 points and is subdivided into 3 subscores: CASS sudomotor (range $0-3$ ), CASS cardiovagal (range $0-3$ ), and CASS adrenergic (range $0-4$ ). Each score has been normalized for the confounding variables of age and gender. ${ }^{24}$ Laboratory evidence of autonomic failure is graded as follows: 0 , absent; $1-3$, mild; $4-6$, moderate; and 7-10, severe failure.

Statistical analysis. Descriptive statistics were used to express and compare continuous and dichotomous results. For continuous measurements we expressed results as means and standard deviations and determined differences between groups using Wilcoxon rank sum test. The Spearman rho tested correlations among continuous variables. For dichotomous variables, we used Fisher exact test. Statistical significance was defined as $p$ value $<0.05$.

RESULTS Of 594 screened patients with potential CIDP, 47 met our clinical and electrophysiologic criteria for classic CIDP and had full autonomic testing performed at Mayo Clinic from 1992 to 2009. All patients had negative hematologic, infectious, endocrine, metabolic, rheumatologic, monoclonal gammopathy, and myeloma screening.

Clinical somatic manifestations. Demographic and neurologic features are summarized in table 1 . Of 47 patients with CIDP identified, 25 were men and 22 were women. On average, onset of symptoms occurred in middle-aged ( $41 \pm 14.9$ years) patients. In the majority of patients, the diagnosis of CIDP was further supported by 1) CSF albuminocytologic dissociation suggesting inflammatory demyelination at nerve root level; and 2) favorable response to immunomodulatory therapy as judged by patient, physician, and neurologic examination.

\section{Table 1 Demographics and disease characteristics of patients with classic CIDP}

\begin{tabular}{|c|c|}
\hline Characteristics & Values \\
\hline Age at onset, $y$, mean $\pm S D$ & $41.4 \pm 15.2$ \\
\hline Male, n/t (\%) & $25 / 47(55)$ \\
\hline Insidious onset, n/t (\%) & $30 / 47(64)$ \\
\hline Progressive course, $\mathrm{n} / \mathrm{t}$ (\%) & $38 / 47(81)$ \\
\hline Motor > sensory, n/t (\%) & $33 / 47(70)$ \\
\hline Immunotherapy responsive, $\mathrm{n} / \mathrm{t}$ (\%) & $38 / 41$ (93) \\
\hline \multicolumn{2}{|l|}{$\operatorname{CSF}(n=45)$} \\
\hline Nucleated cells (cells $/ \mu \mathrm{L}$ ), mean $\pm \mathrm{SD}$ & $3.1 \pm 4.9$ \\
\hline Protein $(\mathrm{mg} / \mathrm{dL})$, mean $\pm \mathrm{SD}$ & $148.1 \pm 146.2$ \\
\hline \multicolumn{2}{|l|}{ At autonomic testing } \\
\hline Age, $y$, mean $\pm S D$ & $45.0 \pm 14.9$ \\
\hline Disease duration, $y$, mean $\pm S D$ & $3.5 \pm 4.3$ \\
\hline \multicolumn{2}{|l|}{ NIS score $(n=46)$} \\
\hline Motor (192 possible points) & $23.9 \pm 25.7$ \\
\hline Deep tendon reflex (20 possible points) & $12.8 \pm 6.9$ \\
\hline Sensory (32 possible points) & $9.8 \pm 9.0$ \\
\hline Total (244 possible points) & $46.5 \pm 32.7$ \\
\hline \multicolumn{2}{|l|}{ QST abnormality of lower limb } \\
\hline $\begin{array}{l}\text { Large fiber abnormality-vibration, } \\
\mathrm{n} / \mathrm{t}(\%)\end{array}$ & 25/36 (69) \\
\hline $\begin{array}{l}\text { Small fiber abnormality-cooling } \\
\text { or heat-pain, } n / t(\%)\end{array}$ & 26/36 (72) \\
\hline
\end{tabular}

Abbreviations: CIDP = chronic inflammatory demyelinating polyradiculoneuropathy; NIS = Neuropathy Impairment Scale; $\mathrm{n} / \mathrm{t}=$ number affected/total number; QST = quantitative sensory testing.

At time of autonomic testing, age was $45.0 \pm$ 14.9 years, duration of illness $3.5 \pm 4.3$ years, and degree of somatic neurologic deficits was moderate (NIS, $46.5 \pm 32.7$ ). The majority of patients had a motor-predominant phenotype of insidious onset and progressive course. Neuropathic pain or sensory complaints were not prominent. Quantitative sensation testing (QST) showed equal proportion of large and small sensory fiber involvement (table 1).

Clinical autonomic manifestations. Autonomic symptoms are summarized in table 2. Autonomic symptoms, as assessed by chart review at time of clinic visit, were reported in $13(23 \%)$ patients at some point during the course of the disease. They preceded neurologic manifestations in $2(4 \%)$ and accompanied neurologic presentation in $4(9 \%)$ patients. The 2 patients with orthostatic intolerance had mild adrenergic abnormalities on autonomic testing. The complaints were mostly mild and transient and were overshadowed by the severity of somatic neurologic manifestations. 


\begin{tabular}{|llll|}
\hline Table 2 & Summary of autonomic symptoms in $\mathbf{4 7}$ patients with classic CIDPa \\
Symptom & $\begin{array}{l}\text { No. (\%) of } \\
\text { patients }\end{array}$ & $\begin{array}{l}\text { No. of patients } \\
\text { with additional } \\
\text { symptoms }\end{array}$ & $\begin{array}{l}\text { Most common } \\
\text { additional symptoms }\end{array}$ \\
Gastrointestinal & $9(19)$ & $5 / 9$ & $\mathrm{GU}$ \\
Genitourinary & $8(17)$ & $7 / 8$ & $\mathrm{GI}$ \\
Secretomotor (sicca) & $3(6)$ & $2 / 3$ & $\mathrm{GU}$ \\
Orthostatic intolerance & $2(4)$ & $2 / 2$ & $\mathrm{GU}$; flushing with sicca \\
Sexual dysfunction & $2(4)$ & $1 / 2$ & $\mathrm{GI}$ \\
Vasomotor (flushing) & $1(2)$ & $1 / 1$ & $\mathrm{OI}$ with sicca \\
Sudomotor (hyperhydrosis) & $1(2)$ & $1 / 1$ & $\mathrm{GI}+\mathrm{GU}$ \\
\hline
\end{tabular}

Abbreviations: $\mathrm{CIDP}=$ chronic inflammatory demyelinating polyradiculoneuropathy; $\mathrm{GI}=$ gastrointestinal; $\mathrm{GU}=$ genitourinary; $\mathrm{Ol}=$ orthostatic intolerance.

a Symptoms derived from standardized chart review.

Upon completion of chart review, we queried The Peripheral Nerve databases to establish which of our 57 patients with classic CIDP had participated in research. We found that $13(28 \%)$ had been enrolled in neuropathy research studies at the time of their initial clinical evaluation. They were required to complete standardized neuropathy questionnaire that included the autonomic symptoms component of the NSC. Four of them reported autonomic symptoms as follows: patient 1: orthostatic intolerance with loss of bladder and rectal control; patient 2: erectile dysfunction; patient 3: anorgasmia; patient 4: dry eyes and mouth.

Autonomic reflex screen and CASS scores. The severity and distribution of autonomic deficits are summarized in table 3. Laboratory autonomic deficits were seen in $22(47 \%)$ patients with total CASS scores $\leq 3$ in all patients indicating that dysautonomia in classic CIDP is of minimal severity and limited distribution. Among those with autonomic deficits, 13 patients had QSART abnormalities that were mostly mild (CASS-sudomotor, $1.5 \pm 0.7$ ), distal or patchy, and involving a single site; 7 had mild cardiovagal abnormalities (CASS-cardiovagal, $1.0 \pm 0.0$ ); and 3 had mild adrenergic abnormalities (CASS-adrenergic $1.0 \pm 0.0$ ) on the Valsalva maneuver as follows: patient 1 , pulse pressure compression, exaggerated early phase II, blunted late phase II, normal head-up tilt, no orthostatic intolerance; patient 2, decreased late phase II and absent phase IV, delayed orthostatic hypotension $(106 / 70 \mathrm{~mm} \mathrm{Hg}$, supine, stable for 5 minutes on HUT, then 68/50 $\mathrm{mm} \mathrm{Hg}$ associated with lightheadedness), but no clear history of orthostatic intolerance; patient 3 , lack of phase IV, normal HUT, and history of orthostatic intolerance.

Thermoregulatory sweat test. Only 8 patients underwent TST, showing heterogeneous results (figure). TST was normal in 3 patients. The remaining 5 had
Table 3 Severity and distribution of autonomic deficits in 47 patients with classic CIDP

$\begin{array}{ll}\text { Autonomic reflex screen abnormalities, } \mathrm{n}(\%) & \\ \text { Total } & 22(47) \\ \text { Sudomotor } & 16(34) \\ \text { Cardiovagal } & 10(21) \\ \text { Adrenergic } & 4(9) \\ \text { CASS score, }{ }^{a} \text { mean } \pm \text { SD } & \\ \text { Sudomotor (0-3) } & 0.5 \pm 0.7 \\ \text { Cardiovagal (0-3) } & 0.2 \pm 0.4 \\ \text { Adrenergic (0-4) } & 0.1 \pm 0.3 \\ \text { Total (0-10) } & 0.8 \pm 0.9 \\ \text { Total CASS } \geq 1, \mathrm{n}(\%) & 22(47) \\ \text { Total CASS } \geq 2, \mathrm{n}(\%) & 12(42) \\ \text { Total CASS } \geq 3, \mathrm{n}(\%) & 2(4) \\ \text { Total CASS } \geq 4, \mathrm{n}(\%) & 0(0)\end{array}$

Abbreviations: CASS $=$ Composite Autonomic Severity Score (range 0-10); CIDP = chronic inflammatory demyelinating polyradiculoneuropathy.

a Maximum CASS scores: sudomotor: 3 = severe widespread anhidrosis; cardiovagal: 3 = blunted cardiovagal responses; adrenergic: 4 = blunted adrenergic response with orthostatic hypotension; total: 10 = severe pandysautonomia.

various degrees of body surface anhidrosis ranging from $2 \%$ to $59 \%$ (mean $12.8 \% \pm 21.0 \%$ ) with various patterns of distribution: distal in 2, regional (mainly lower body) in 2, and mixed (distal and patchy) in 1 patient. Sites with reduced QSART were mostly located in areas of intact TST sweating (indicating a quantitative impairment) and to a lesser degree in some areas with TST anhidrosis, a combination supporting postganglionic site of sudomotor denervation. There were no sites of normal QSART located in areas of TST anhidrosis to indicate a preganglionic lesion. Altogether, these findings suggest that sudomotor abnormalities were essentially mostly distal and postganglionic (figure).

Relation to somatic features. There was no correlation between severity and distribution of autonomic deficits (CASS) and severity of somatic deficits (NIS) $(r=-0.073, p=0.628)$ or disease duration $(r=$ $0.166, p=0.265)$. There was no association between presence of QST-small fiber abnormalities and definite autonomic deficits defined as CASS $\geq 2(p<$ $0.69)$. There was no association between gender and CASS $\geq 2(p<0.33)$. Age at disease onset $(50.5 \pm$ 13.0 vs $38.3 \pm 14.8 ; p<0.018)$ and at autonomic testing $(55.0 \pm 14.1$ vs $41.5 \pm 13.7 ; p<0.012)$ was significantly higher in patients with a total CASS $\geq 2$.

DISCUSSION In this study, we have characterized the features of autonomic dysfunction in classic CIDP as follows: 1) autonomic symptoms were 


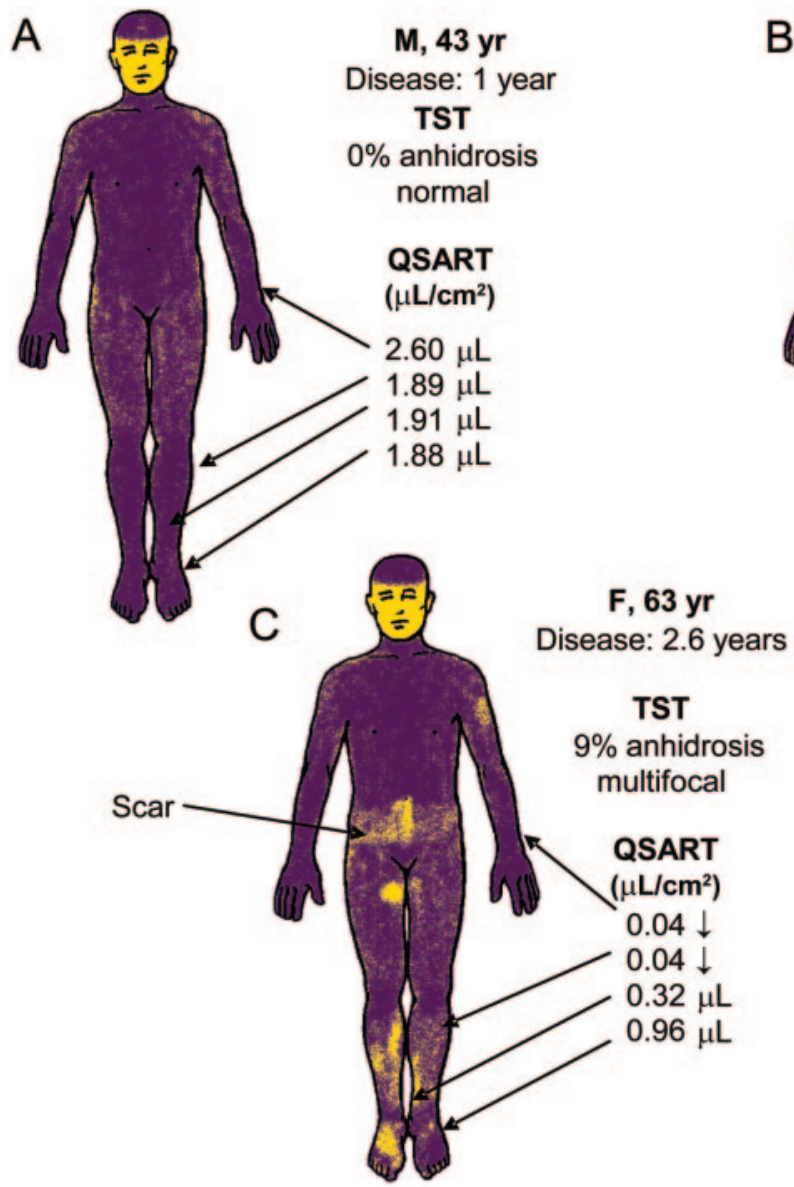

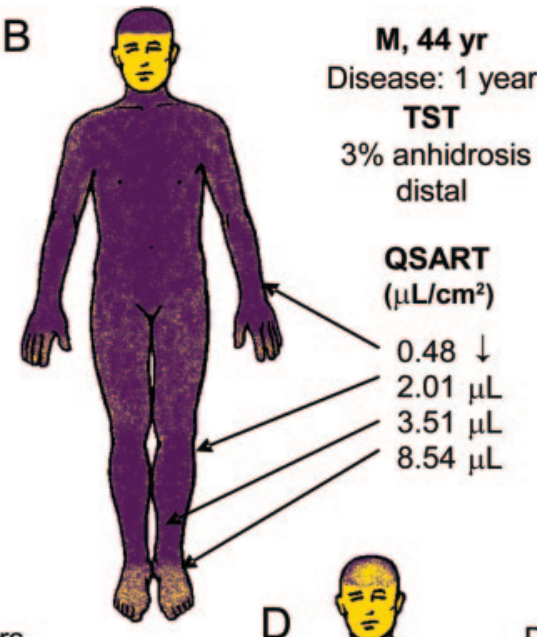

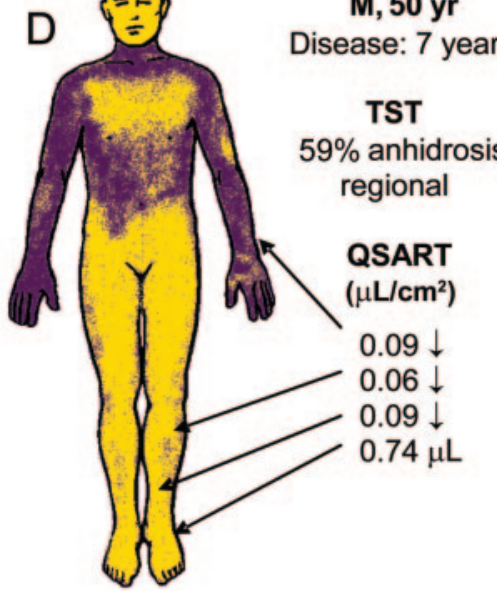

(A) Normal thermoregulatory sweat test (TST) and quantitative sudomotor axon reflex testing (QSART). (B) Mild distal anhidrosis with a focal postganglionic lesion (forearm). (C) Mild anhidrosis in a multifocal pattern with postganglionic (forearm and proximal leg) lesions. (D) Moderate TST anhidrosis in a regional pattern consistent with a polyradicular distribution with concomitant postganglionic involvement (polyradiculoneuropathy).

sparse and when present mainly manifested as bowel and bladder complaints; 2) autonomic deficits were minor and limited to sympathetic sudomotor and cardiovagal function with relative sparing of sympathetic adrenergic function; 3) the lesion appeared to be mainly distal and postganglionic; and 4) autonomic deficits did not relate to somatic deficits. This distinctive autonomic profile may have diagnostic implications and can be compared in a standard way to other neuropathies that have been studied using CASS as summarized in table 4. In general, the table shows that autonomic findings in CIDP are minor compared to other forms of neuropathy, especially other inflammatory neuropathies. ${ }^{25-34}$

The current study is limited by 1 ) its retrospective nature with potential bias to patient recall and examiner questions; and 2) potential selection bias to more dysautonomia in those referred for autonomic testing. However, selection bias for autonomic assessment is unlikely because autonomic testing is a routine component of neuropathy evaluation at Mayo Clinic peripheral neuropathy clinic. Furthermore, if selection bias existed, the degree of autonomic neuropathy in CIDP would even be milder and more limited than our estimation.

The lack of serious clinical dysautonomia in our series is in keeping with the majority of previous reports that show that autonomic symptoms are usually mild in CIDP. 5, 7,35-37 Conversely, deficits were frequently subclinical as previously reported. ${ }^{1} \mathrm{Al}-$ though genitourinary symptoms were frequent, pure (pudendal) somatic dysfunction could potentially account for micturition complaints. ${ }^{4}$

The profile of autonomic laboratory deficits (CASS) found in our patients is in agreement with most studies, specifically the relatively intact baroreflex-mediated peripheral vasoconstriction and paucity of florid orthostatic hypotension in the majority of patients. ${ }^{1,2,10}$ Only 3 patients had minimal adrenergic impairment based on the blood pressure profile on the Valsalva maneuver and only one of them showed delayed orthostatic hypotension on HUT. Higher frequency and severity of sympathetic adrenergic failure has been reported on moderately 
Table 4 Comparison of CASS scores between classic CIDP and other neuropathies previously reported in the literature

\begin{tabular}{|c|c|c|c|c|c|}
\hline \multirow[b]{2}{*}{ Neuropathic conditions } & \multicolumn{5}{|c|}{ CASS total and severity distribution } \\
\hline & $\begin{array}{l}\text { Mean or } \\
\text { median }\end{array}$ & $0, \%$ & $(1-3), \%$ & $\begin{array}{l}(4-6), \\
\%\end{array}$ & $\begin{array}{l}(7-10), \\
\%\end{array}$ \\
\hline Classic CIDP (current study), mean $(n=47)$ & 0.8 & 53 & 47 & 0 & 0 \\
\hline Community diabetes, ${ }^{25}$ mean $(n=202)$ & 2.3 & NA & NA & NA & NA \\
\hline Small-fiber neuropathy, ${ }^{26}$ mean $(n=125)$ & 2.7 & 48 & 24 & 27 & 1 \\
\hline Sicca complex-neuropathy, ${ }^{29}(n=34)$ & NA & 15 & 38 & 35 & 12 \\
\hline Nondiabetic LRPN, ${ }^{31}$ median $(n=12)$ & 5.0 & 8 & 8 & 50 & 33 \\
\hline Painless diabetic LRPN, ${ }^{32}$ median $(n=9)$ & 6.0 & NA & NA & NA & NA \\
\hline $\begin{array}{l}\text { Autoimmune autonomic ganglionopathy, }{ }^{27} \\
\text { mean }(n=27)\end{array}$ & 6.0 & 0 & 26 & 11 & 63 \\
\hline Amyloid neuropathy, ${ }^{28}$ mean $(n=58)$ & 6.7 & 0 & 10 & 42 & 48 \\
\hline TTR-amyloid neuropathy, ${ }^{33}$ median $(n=36)$ & 7.0 & 17 & 11 & 14 & 58 \\
\hline Diabetic LRPN, ${ }^{30}$ median $(n=14)$ & 7.0 & 0 & 29 & 14 & 57 \\
\hline $\begin{array}{l}\text { Subacute diabetic proximal neuropathy, }{ }^{34} \\
\text { mean }(n=28)\end{array}$ & 7.8 & NA & NA & NA & 75 \\
\hline
\end{tabular}

Abbreviations: CASS = Composite Autonomic Severity Score (range 0-10); CIDP = chronic inflammatory demyelinating polyradiculoneuropathy; LRPN = lumbosacral radiculoplexus neuropathy; $\mathrm{NA}=$ not available; TTR $=$ transthyretin.

disabled patients based purely on abnormalities on prolonged head-up tilt. ${ }^{3}$ Without correlation with more solid indices of adrenergic function such as the blood pressure profile to the Valsalva maneuver, a nonadrenergic cause such as hypovolemia and deconditioning cannot be ruled out.

Our TST findings, like those previously reported, ${ }^{1}$ were heterogeneous. Combining both studies, abnormalities range from normal to various degrees of anhidrosis and distribution patterns including distal, focal (patchy on limbs), regional, and global. Although the site of sudomotor lesion appeared predominantly postganglionic as a result of lesions at the distal axon, larger samples of patients with TST are needed to corroborate these findings. If correct, they likely represent summated distal axonal dysfunction in different body distributions.

A general agreement between loss of autonomic fibers and loss of somatic small fibers has previously been reported. ${ }^{38}$ Contrary to our hypothesis, we did not find such association. Moreover, autonomic features were independent of neurologic disease duration and severity. These findings may suggest different pathogenic mechanisms between somatic and autonomic fiber loss in patients with CIDP.

Our data characterize the autonomic dysfunction in CIDP predominantly as a mild, limited, and distal postganglionic cholinergic neuropathy. Future directions should explore the utility of CASS in differentiating conditions such as acute-onset CIDP from acute inflammatory demyelinating polyradiculoneuropathy (AIDP) or diabetes with CIDP from diabetic lumbosacral radiculoplexus neuropathy (DLRPN). In AIDP, autonomic symptoms and deficits are frequent and sometimes severe. In contrast, we have shown that CIDP dysautonomia is limited and minor. DLRPN is frequently associated with early and well-controlled diabetes. It has previously been shown that autonomic neuropathy in average community diabetic patients is mild and limited. Hence, mild dysautonomia in diabetes with CIDP is predicted. In contrast, DLRPN is associated with frequent, severe, and widespread autonomic symptoms and deficits. Moderate to severe autonomic deficits in a more widespread distribution (CASS $\geq 4$ ) were not found in our CIDP series. When present, concern for an alternative diagnosis should be raised.

\section{AUTHOR CONTRIBUTIONS}

Dr. Figueroa: design, draft, statistical analysis, interpretation of data. Dr. P.J.B. Dyck: design, draft, editing. Dr. Laughlin: contributed content and editing. Dr. Mercado: interpretation of data, revision. Dr. Massie: data collection, analysis. Dr. Sandroni: data interpretation, editing. Dr. P.J. Dyck: conception, analysis. Dr. Low: conception, design, draft, analysis, funding.

\section{STUDY FUNDING}

Supported in part by National Institutes of Health (NS 32352 Autonomic Disorders Program Project, NS 44233 Pathogenesis and Diagnosis of Multiple System Atrophy, U54 NS065736 Autonomic Rare Disease Clinical Consortium), Mayo CTSA (UL1 RR24150), NIH Research Training Grant T32 HD07447, and Mayo Funds to Dr. Low. The Autonomic Diseases Consortium is a part of the NIH Rare Diseases Clinical Research Network (RDCRN). Funding or programmatic support for this project has been provided by U54 NS065736 from the National Institute of Neurological Diseases and Stroke (NINDS) and the NIH Office of Rare Diseases Research (ORDR). The content is solely the responsibility of the authors and does not necessarily represent the official views of the National Institute of Neurological Disorders and Stroke or the National Institutes of Health.

\section{DISCLOSURE}

Dr. Figueroa, Dr. P.J.B. Dyck, Dr. Laughlin, and Dr. Mercado report no disclosures. Dr. Massie has received publishing royalties from UpToDate, Inc. Dr. Sandroni reports no disclosures. Dr. P.J. Dyck serves as an Associate Editor for Diabetes and receives research support from the NIH/ NINDS. Dr. Low serves as a Clinical Editor for Autonomic Neuroscience, receives research support from NIH/NINDS. and is a consultant for WR Medical, Stillwater, MN.

Received June 14, 2011. Accepted in final form October 26, 2011.

\section{REFERENCES}

1. Ingall TJ, McLeod JG, Tamura N. Autonomic function and unmyelinated fibers in chronic inflammatory demyelinating polyradiculoneuropathy. Muscle Nerve 1990;13: $70-76$.

2. Lyu RK, Tang LM, Wu YR, Chen ST. Cardiovascular autonomic function and sympathetic skin response in chronic inflammatory demyelinating polyradiculoneuropathy. Muscle Nerve 2002;26:669-672.

3. Stamboulis E, Katsaros N, Koutsis G, Iakovidou H, Giannakopoulou A, Simintzi I. Clinical and subclinical autonomic dysfunction in chronic inflammatory demyelinating polyradiculoneuropathy. Muscle Nerve 2006;33:78-84. 
4. Sakakibara R, Hattori T, Kuwabara S, Yamanishi T, Yasuda K. Micturitional disturbance in patients with chronic inflammatory demyelinating polyneuropathy. Neurology 1998;50:1179-1182.

5. Dyck PJ, Lais AC, Ohta M, Bastron JA, Okazaki H, Groover RV. Chronic inflammatory polyradiculoneuropathy. Mayo Clin Proc 1975;50:621-637.

6. Prineas JW, McLeod JG. Chronic relapsing polyneuritis. J Neurol Sci 1976;27:427-458.

7. McCombe PA, Pollard JD, McLeod JG. Chronic inflammatory demyelinating polyradiculoneuropathy: a clinical and electrophysiological study of 92 cases. Brain 1987; 110:1617-1630

8. Boukhris S, Magy L, Li Y, Debras C, Vallat JM. [Autonomic nervous system involvement in chronic inflammatory demyelinating polyneuropathy.] Rev Neurol 2005; 161:1228-1231.

9. Ishii K, Tamaoka A, Fujita Y, Shoji S. Bladder and bowel dysfunction in chronic inflammatory demyelinating polyradiculoneuropathy. Eur J Intern Med 2005;16:211-213.

10. Yamamoto K, Watarai M, Hashimoto T, Ikeda S. Chronic inflammatory demyelinating polyradiculoneuropathy with autonomic involvement. Muscle Nerve 2005;31:108-112.

11. Hantson P, Kevers L, Fabien N, Van Den Bergh P. Acuteonset chronic inflammatory demyelinating polyneuropathy with cranial nerve involvement, dysautonomia, respiratory failure, and autoantibodies. Muscle Nerve 2010;41:423-426.

12. Low PA. Composite autonomic scoring scale for laboratory quantification of generalized autonomic failure. Mayo Clin Proc 1993;68:748-752.

13. Laughlin RS, Dyck PJ, Melton LJ, 3rd, Leibson C, Ransom J, Dyck PJB. Incidence and prevalence of CIDP and the association of diabetes mellitus. Neurology 2009;73:39-45.

14. Research criteria for diagnosis of chronic inflammatory demyelinating polyneuropathy (CIDP): report from an Ad Hoc Subcommittee of the American Academy of Neurology AIDS Task Force. Neurology 1991;41:617-618.

15. European Federation of Neurological Societies/Peripheral Nerve Society Guideline on management of chronic inflammatory demyelinating polyradiculoneuropathy: report of a joint task force of the European Federation of Neurological Societies and the Peripheral Nerve Society. J Periph Nerv Syst 2005;10:220-228.

16. Dyck PJ, Sherman WR, Hallcher LM, et al. Human diabetic endoneurial sorbitol, fructose, and myo-inositol related to sural nerve morphometry. Ann Neurol 1980;8: 590-596.

17. Dyck PJ, Zimmerman IR, O'Brien PC, et al. Introduction of automated systems to evaluate touch-pressure, vibration, and thermal cutaneous sensation in man. Ann Neurol 1978;4:502-510.

18. Dyck PJ, Zimmerman I, Gillen DA, Johnson D, Karnes JL, O'Brien PC. Cool, warm, and heat-pain detection thresholds: testing methods and inferences about anatomic distribution of receptors. Neurology 1993;43:1500-1508.

19. Dyck PJ, Turner DW, Davies JL, O'Brien PC, Dyck PJB, Rask CA. Electronic case-report forms of symptoms and impairments of peripheral neuropathy. Can J Neurol Sci 2002;29:258-266.

20. Low PA. Autonomic nervous system function. J Clin Neurophysiol 1993;10:14-27.
21. Low PA, Caskey PE, Tuck RR, Fealey RD, Dyck PJ. Quantitative sudomotor axon reflex test in normal and neuropathic subjects. Ann Neurol 1983;14:573-580.

22. Fealey RD, Low PA, Thomas JE. Thermoregulatory sweating abnormalities in diabetes mellitus. Mayo Clin Proc 1989;64:617-628.

23. Low PA. Evaluation of sudomotor function. Clin Neurophysiol 2004;115:1506-1513.

24. Low PA, Denq JC, Opfer-Gehrking TL, Dyck PJ, O’Brien PC, Slezak JM. Effect of age and gender on sudomotor and cardiovagal function and blood pressure response to tilt in normal subjects. Muscle Nerve 1997;20:1561-1568.

25. Low PA, Benrud-Larson LM, Sletten DM, et al. Autonomic symptoms and diabetic neuropathy: a populationbased study. Diabetes Care 2004;27:2942-2947.

26. Low VA, Sandroni P, Fealey RD, Low PA. Detection of small-fiber neuropathy by sudomotor testing. Muscle Nerve 2006;34:57-61.

27. Suarez GA, Fealey RD, Camilleri M, Low PA. Idiopathic autonomic neuropathy: clinical, neurophysiologic, and follow-up studies on 27 patients. Neurology 1994;44: 1675-1682.

28. Wang AK, Fealey RD, Gehrking TL, Low PA. Patterns of neuropathy and autonomic failure in patients with amyloidosis. Mayo Clin Proc 2008;83:1226-1230.

29. Wright RA, Grant IA, Low PA. Autonomic neuropathy associated with sicca complex. J Auton Nerv Syst 1999;75: $70-76$.

30. Dyck PJB, Norell JE, Dyck PJ. Microvasculitis and ischemia in diabetic lumbosacral radiculoplexus neuropathy. Neurology 1999;53:2113-2121.

31. Dyck PJB, Norell JE, Dyck PJ. Non-diabetic lumbosacral radiculoplexus neuropathy: natural history, outcome and comparison with the diabetic variety. Brain 2001;124: 1197-1207.

32. Garces-Sanchez M, Laughlin RS, Dyck PJ, Engelstad JK, Norell JE, Dyck PJB. Painless diabetic motor neuropathy: a variant of diabetic lumbosacral radiculoplexus: neuropathy? Ann Neurol Epub 2010.

33. Kim DH, Zeldenrust SR, Low PA, Dyck PJ. Quantitative sensation and autonomic test abnormalities in transthyretin amyloidosis polyneuropathy. Muscle Nerve 2009;40: 363-370.

34. Pascoe MK, Low PA, Windebank AJ, Litchy WJ. Subacute diabetic proximal neuropathy. Mayo Clin Proc 1997;72: 1123-1132.

35. Barohn RJ, Kissel JT, Warmolts JR, Mendell JR. Chronic inflammatory demyelinating polyradiculoneuropathy: clinical characteristics, course, and recommendations for diagnostic criteria. Arch Neurol 1989;46:878-884.

36. Gorson KC, Allam G, Ropper AH. Chronic inflammatory demyelinating polyneuropathy: clinical features and response to treatment in 67 consecutive patients with and without a monoclonal gammopathy. Neurology 1997;48:321-328.

37. Simmons Z, Albers JW, Bromberg MB, Feldman EL. Presentation and initial clinical course in patients with chronic inflammatory demyelinating polyradiculoneuropathy: comparison of patients without and with monoclonal gammopathy. Neurology 1993;43:2202-2209.

38. Singer W, Spies JM, McArthur J, et al. Prospective evaluation of somatic and autonomic small fibers in selected autonomic neuropathies. Neurology 2004;62:612-618. 